\title{
Lidil
}

Revue de linguistique et de didactique des langues

$57 \mid 2018$

Démarches créatives, détours artistiques et appropriation des langues

\section{Démarches créatives en DDdL : créativité ou poïesis ?}

Creative Approaches in Language Teaching: Creativity or Poïesis?

\section{Emmanuelle Huver et Joanna Lorilleux}

\section{(2) OpenEdition}

Journals

Édition électronique

URL : http://journals.openedition.org/lidil/4885

DOI : $10.4000 /$ lidil. 4885

ISSN : 1960-6052

Éditeur

UGA Éditions/Université Grenoble Alpes

Édition imprimée

ISBN : 978-2-37747-048-8

ISSN : $1146-6480$

Référence électronique

Emmanuelle Huver et Joanna Lorilleux, « Démarches créatives en DDdL : créativité ou poïesis ? », Lidil [En ligne], 57 | 2018, mis en ligne le 01 mai 2018, consulté le 02 mai 2019. URL : http:// journals.openedition.org/lidil/4885 ; DOI : 10.4000/lidil.4885

Ce document a été généré automatiquement le 2 mai 2019.

(c) Lidil 


\title{
Démarches créatives en DDdL : créativité ou poïesis?
}

Creative Approaches in Language Teaching: Creativity or Poïesis?

\author{
Emmanuelle Huver et Joanna Lorilleux
}

\section{Introduction}

1 La question de l'articulation entre art et didactique des langues n'est pas nouvelle, mais sa caractérisation par le terme de créativité est plus récente. Une des visées de cet article est donc de s'interroger sur ce terme en DDdL, pour mettre en débat, par la notion de poïsis, des bases épistémologiques selon nous très unanimes.

Dans un article précédent (Castellotti, Debono \& Huver, 2017), nous avons traité de l'histoire de la notion de créativité et de son évolution. Pour résumer, nous avions mis en évidence que la rencontre entre innovation et créativité a très largement participé à inscrire la créativité dans un imaginaire aujourd'hui encore prévalent caractérisé par: l'injonction à la nouveauté opposée aux traditions, sa biologisation, sa technicisation, une logique de production.

3 Nous avons souhaité approfondir ici cette réflexion, en nous focalisant sur les conceptualisations de la créativité en DDdL. Pour ce faire, nous nous sommes appuyées sur quelques textes récents considérés comme des références et y avons relevé les caractéristiques définitionnelles récurrentes de la créativité, qui se sont avéré largement reprendre les tendances générales évoquées ci-avant. En regard de ces définitions, nous avons souhaité proposer, dans un second temps, une conceptualisation de la notion qui s'enracine dans un tout autre univers de référence, d'ordre phénoménologique herméneutique $(\mathrm{PH})$. La thèse que nous défendons dans ce texte part de l'idée que la distinction entre poïsis et créativité s'ancre dans la controverse fondatrice en philosophie entre $\mathrm{PH}$ et philosophie analytique, notamment pour ce qui concerne la philosophie du langage. Il s'agit ainsi de mettre en débat les épistémologies sous-jacentes aux conceptions usuelles de la créativité, démarche selon nous nécessaire pour (ré)ouvrir 
des possibilités de réflexion et donc de choix dans ce qui apparait souvent, sinon, comme des évidences sans alternatives. Dans une dernière partie, nous évoquerons les implications et les enjeux de cette réflexion pour la DDdL, en posant l'expérience artistique comme mode d'appropriation, au sens de Castellotti (2017), et donnerons quelques exemples d'intervention.

\section{La notion de créativité en DDdL : définitions et implications}

4 Nous commencerons cette réflexion en nous intéressant aux conceptualisations les plus répandues et (donc) les plus dominantes de la notion de créativité en DDdL. Il s'agira, ce faisant, de mettre en évidence les univers théoriques et philosophiques qui les fondent et les implications qui en découlent.

\subsection{Repères définitionnels en DDdL}

Les articles définitoires considérés font tout d'abord apparaitre un certain nombre de traits transversalement récurrents, qui témoignent de tendances fortes et notablement similaires aux tendances générales évoquées en introduction. On retiendra notamment :

- le lien fort (voire l'indistinction) entre créativité et innovation (cf. par exemple Csikszentmihalyi, 2006, cité par Piccardo, 2016, p. 56);

- la caractérisation de la créativité au moyen de deux critères: la "nouveauté » et "l'adaptation» (au contexte dans lequel elle se manifeste), caractérisation opérée par Lubart (2003) et très largement reprise dans les travaux considérés, au point même que Botella, Nelson et Zenasni (2016) affirment (sans plus développer) que «sans ces deux caractéristiques, chaque idée un peu fantaisiste ou anticonformiste serait créative » (p. 33).

Ces récurrences fortes laissent aussi apparaitre des angles morts, notamment le fait qu'aucun des travaux considérés, bien qu'ils s'inscrivent en DDdL, ne thématise la spécificité qu'il y aurait (ou non) à envisager la créativité dans/par les langues :

- ni au plan sociolinguistique, où la question de la créativité linguistique a pourtant donné lieu à de houleux débats autour de la norme ${ }^{1}$;

- ni au plan linguistique, alors que la créativité linguistique constitue une notion bien connue, voire incontournable, dans les sciences du langage.

7 La seule mention à la créativité linguistique que nous ayons trouvée est proposée par Aden (2009) :

Parler est en soi un acte créatif puisque nous varions à l'infini notre discours au moyen d'un nombre fini de mots et de structures dans des situations jamais identiques. (p. 174)

Cette citation ne comporte pas de référence explicite, mais renvoie manifestement, en filigrane, à la définition chomskyenne de la créativité linguistique :

A person who knows a language has mastered a system of rules that assigns sound and meaning in a definite way for an infinite class of possible sentences ${ }^{2}$.

A mature speaker can produce a new sentence of his language on the appropriate occasion and others speakers can understand it immediately, though it is equally new to them. Most of our linguistic experience, both as speakers and hearers, is with new sentences; once we have mastered a language, the class of sentences with which we can operate fluently and without difficulty or hesitation is so vast that for all practical purposes [...] we may regard it as infinite ${ }^{3}$. 
Il est aussi intéressant de relever le fait que Chomsky (1982) définit la créativité linguistique par les critères de nouveauté et d'appropriété, également thématisés par Lubart dans le domaine de la psychologie, même si les circulations et les (probables ?) héritages restent implicites voire tus ${ }^{4}$. Outre ce point de convergence, d'autres transversalités méritent d'être relevées.

\subsection{Transversalités}

9 Pour définir la créativité, Capron Puozzo (2016) parle de " capharnaüm terminologique » (p.15). Cependant, sous cette dite hétérogénéité, il nous semble que les choix épistémologiques que nous allons relever ici sont remarquablement continus, stables et récurrents.

\subsubsection{Production}

Les différentes définitions de la créativité proposées en DDdL dans les ouvrages consultés renvoient toutes à une définition clairement productiviste de la notion. Capron Puozzo (2016) indique ainsi que la pensée créative devient créativité "quand elle se matérialise dans une production ou une performance »(p.18) et qu'une pédagogie de la créativité vise à "concevoir et mettre en œuvre des dispositifs d'enseignement/ apprentissage qui favorisent des processus et des performances créatifs pour une démarche de production » (p. 24). De même, Aden (2009), dans une réflexion insistant sur le possible sentiment de très forte contrainte lié à la «la rigueur et [au] temps de l'élaboration d'une œuvre artistique », affirme que « le partage avec un public transcende le souvenir de la contrainte»; elle en tire la conclusion qu'il est "absolument fondamental que toute démarche artistique se concrétise par une présentation de l'œuvre » (p. 178), par une production, donc.

11 Cette idée d'une créativité se réalisant prioritairement dans la production se retrouve également chez Chomsky; c'est en tout cas la thèse que défend, de manière convaincante, Joseph (2004 et 2011):

Ainsi, la créativité linguistique que N. Chomsky appelle « infinie » ne l'est que du côté du sujet parlant. Normalement, l'interprétation par l'auditeur est limitée et, dans les autres cas, là où l'auditeur fait quelque chose de créatif, N. Chomsky préfère l'appeler alors imposition.

Le caractère péjoratif du terme imposition constitue ainsi, selon Joseph (2011, p. 58-59), un moyen de délégitimer l'activité d'interprétation et, donc, de réception, et, ce faisant, de justifier, dans les recherches sur la créativité linguistique, l'attention exclusivement accordée au pôle de la production.

\subsubsection{Utilité}

12 Les nombreux travaux consultés, lorsqu'ils argumentent le recours à une pédagogie de la créativité, présentent celle-ci comme indispensable à la réussite individuelle, mais aussi économique et sociale (Piccardo, 2016) et permettant de créer « une relation efficace avec le spectateur » (Sofia, 2016, p. 96 ; elle souligne). La créativité est ainsi d'abord considérée comme un moyen d'adapter les publics (généralement, dans ces travaux, des publics scolaires) aux « caractéristiques personnelles attendues chez l'employé d'aujourd'hui qui n'est plus uniquement un professionnel compétent, mais un professionnel qui se doit 
d'innover pour s'adapter aux besoins de la société » (Puozzo Capron, 2014, p. 102). Dans ces citations, l'efficacité et l'utilité (sociale ?) sont ainsi essentiellement conçues comme de nécessaires " adaptations aux besoins ». Certains n'hésitent pas, d'ailleurs, à franchir le pas vers une forme de consumérisme explicite et assumé : Gardner (cité par Capron Puozzo, 2016) n'indique-t-il pas qu'« en fin de compte, il ne reste plus à ces créations qu'à se faire accepter par des consommateurs avertis » (p.17)?

En outre, l'adaptation aux besoins n'est parfois pas très loin de la soumission à ceux-ci. C'est pourquoi, au-delà de la critique économiciste, l'idée même de considérer l'adaptation au contexte comme un des piliers définitoires de la notion doit aussi impérativement être questionnée. Il ne s'agit plus, alors, d'interroger la demande (institutionnelle notamment) de créativité, mais bien la manière dont les notions sont conceptualisées, au plan didactique plus particulièrement. La langue, par exemple, reste avant tout conçue comme un outil de communication - la créativité constituant alors un " détour " (ou un prétexte?) pour favoriser cette communication, voire dans certains cas plus ou moins caricaturaux, faire passer la " pilule amère de la grammaire ", de manière dite «ludique». Ce qui est alors empêché, lorsque la langue - et la créativité - sont conceptualisées sous l'angle de l'utilité, de l'efficacité et de l'adaptation, c'est de les penser, à l'inverse, comme profondément subversives, malgré ce qui a pu être développé à ce sujet en philosophie et malgré de nombreux exemples significatifs dans l'histoire des arts.

\subsubsection{Cognitivisme}

Les travaux consultés s'inscrivent, dans leur immense majorité, dans un cadre théorique cognitiviste ${ }^{5}$. Un premier ensemble de ces travaux considère que la créativité est avant tout à comprendre comme une activité du cerveau. Guilford, fondateur de cette conception de la créativité (et abondamment cité dans les différents travaux de DDdL sur le sujet), est en effet présenté comme ayant développé cette notion en réaction aux behavioristes, qui n'avaient pas d'intérêt " pour ce qui se passait au niveau du cerveau " (Piccardo, 2016, p. 52).

15 Cette conception "cérébro-centrée " de la créativité est étendue au corps dans son ensemble, dans un deuxième ensemble de travaux mobilisant la notion d'« embodied creativity» (Sofia, 2016; Aden, 2009). Même si elle ne se limite pas à la relation entre créativité et aires du cerveau, cette théorisation reste d'ordre neurobiologique/ physiologique. Cette naturalisation de la créativité est également défendue par Chomsky par ailleurs, puisque selon lui, la créativité est un attribut humain inné. Enfin, il faut relever que les métaphores mobilisées dans ces travaux sont révélatrices de cette conception prioritairement biologisante de la créativité, comme par exemple chez Piccardo (2016, citant Csikszentmihaly), où la créativité est explicitement comparée à un processus biologique, neurochimique :

La créativité est l'équivalent culturel du processus de modification génétique responsable de l'évolution biologique où des développements aléatoires se produisent à notre insu dans la chimie de nos chromosomes. (p. 57) 


\subsection{Implications}

16 Ces transversalités fortes ne sont pas sans conséquences. Elles permettent certes de penser une créativité "démocratisée», en ce que nous sommes tous mentalement équipés d'un potentiel créatif illimité (notamment au plan langagier) :

Les opinions de Chomsky sur la créativité ont connu un succès énorme [...] dans tout le Zeitgeist des années 60. L'implication était que tout être humain est infiniment créatif, dès l'enfance. De ce fait, la créativité ne serait pas limitée à ces «créateurs» intellectuels que personne n'aime vraiment dans les cultures anglophones: les gens de gauche les associent à la décadence bourgeoise, les gens de droite au socialisme. (Joseph, 2011, p. 57)

Mais, ce faisant, elles réduisent aussi la créativité à un processus d'auto-expression de son intériorité subjective/psychique par un individu considéré comme une entité autonome, une créativité en définitive soustraite au social, au culturel, à l'historique.

Dès lors qu'elle vise l'adaptation et/ou qu'elle est biologisée, la créativité se trouve ainsi à la fois :

- dépolitisée ${ }^{6}$, comme l'indique la citation de Joseph ci-dessus, et comme le suggère également Varela, qui avait lui-même explicitement fait part de ses fortes préventions quant à une " application » au plan social de ses travaux en biologie :

Je me refuse à appliquer l'auto-poièse au plan social. Cela peut vous surprendre mais je m'y refuse pour des raisons politiques. L'histoire a montré que la biologie holistique est fort intéressante, mais elle a toujours eu aussi sa part d'ombre; chaque fois qu'elle s'est laissée aller à l'application au modèle social, il y a eu des glissements vers le fascisme et d'autres positions autoritaires comme l'eugénisme. (Varela, entretien dans Benkirane, 2006, p. 155)

- et technicisée, ou pour le dire autrement, « dépoïétisée » (terme sur lequel nous reviendrons dans notre troisième partie): les épistémologies de référence de ces travaux s'inscrivent en effet toutes, plus ou moins directement et au moins au vu des théories de référence qu'ils convoquent, dans une conception expérimentale de la science, qui vise à objectiver et expliquer (exhaustivement) la créativité. Lubart (2003) (de même que Botella, Nelson \& Zenasni, 2016, par exemple) a ainsi recours à la psychométrie, aux statistiques et aux protocoles expérimentaux, dans l'objectif de «mesurer le potentiel créatif » des individus ; Piccardo (2016) se donne quant à elle pour projet de « donner une explication complète de la créativité » (p. 53).

La conjonction de ces orientations productiviste, utilitaire et cognitiviste ont alors pour conséquence (et aussi souvent comme visée) de rendre la créativité contrôlable. Ainsi, Joseph montre bien que la créativité de Chomsky est une créativité qui obéit à des règles, lesquelles permettent donc, de fait, d'exercer des formes de contrôle. De même, Botella, Nelson et Zenasni (2016) proposent de dégager différentes étapes dans le processus créatif, ce qui permet d'en déduire des procédures, à transposer ensuite en dispositifs formatifs. La boucle didactique est alors bouclée lorsque des préconisations sont formulées pour évaluer la créativité : Capron Puozzo (2015) propose ainsi de transposer les critères définitionnels de Lubart en critères d'évaluation : une production serait alors évaluée comme effectivement créative dès lors qu'elle serait « nouvelle » ET « adaptée ».

Rendre la créativité contrôlable, donc... Mais aussi contrôlante, comme nous l'évoquions plus haut à propos de la porosité entre adaptation et soumission : la focalisation sur la production permet en effet de faciliter le processus évaluatif, en l'appuyant sur des traces 
rapportées à des critères, en l'occurrence, notamment, les critères d'adaptation et de nouveauté. Le critère d'adaptation devient ainsi, d'une certaine manière, un véhicule du contrôle, non seulement de la norme (socio)linguistique (il faut produire des énoncés grammaticalement et/ou pragmatiquement adaptés), mais aussi de la norme sociale, puisqu'il s'agit d'agir, de se comporter, de manière adaptée, par exemple à ce qu'attend l'école, ou la société, ou l'interlocuteur dans l'interaction.

21 Au-delà du fait de constater ces tendances, et parce qu'elles dessinent implicitement une certaine conception de l'homme et de la société (et c'est bien ce caractère implicite qui nous pose problème, puisqu'il occulte toute possibilité de débat), il nous semble nécessaire de les interroger, en inscrivant la créativité dans un autre univers de références allant puiser à d'autres sources théoriques, épistémologiques, philosophiques. C'est ce que nous nous proposons de faire ci-après.

\section{Poïesis : un autre univers de références pour la créativité en DDdL}

C'est à elle [cette puissance propre à la parole] qu'est sensible le poète, qui sait que le langage n'est pas seulement destiné à la communication, qu'il n'est pas seulement un moyen inventé par les hommes pour se comprendre les uns les autres, mais qu'il a aussi le pouvoir de faire surgir le monde devant nos yeux. (Dastur, 2014, p. 56)

Dans l'optique de pluraliser ce qui semble souvent (et implicitement) faire consensus autour de la notion de créativité en DDdL, il nous semble nécessaire de commencer par revenir sur une des dimensions fondamentales de cette discipline, à savoir le langage et les langues. Nous défendrons ici l'idée que le fait d'envisager le langage et les langues prioritairement comme poïesis, c'est-à-dire comme expérience nécessairement créative du monde, bouleverse les priorités de la didactique, en orientant ses questionnements vers la réception et la compréhension (au sens gadamérien du terme, sur lequel nous reviendrons) plutôt que vers la production; vers la relation qui peut s'y nouer plutôt que vers l'efficacité des dispositifs à mettre en place; vers une appréhension culturelle et historiale de l'humain plutôt que vers une conception essentiellement biologique du «Vivant» (cf. Aden, 2009, par exemple).

\subsection{Langage et langues comme expériences du monde}

Dans les courants $\mathrm{PH}^{7}$, le langage et les langues sont considérés comme expériences du monde, et ce, sous plusieurs angles complémentaires.

En premier lieu vient l'idée selon laquelle le langage se détache, pour chacun, singulièrement, "d'un ordre pré-linguistique », que les phénoménologues (de Husserl à Romano, en passant par Merleau-Ponty) nomment antéprédicatif, un ordre lié à la perception première, sensible, que nous avons des phénomènes.

En second lieu, le langage et la pensée (en tant qu'«accès » au monde) sont envisagés comme consubstantiels. Ainsi, selon Trabant (2014) :

Le langage n'[...]est pas seulement considéré comme une technique secondaire pour la communication de la pensée. Il est la production de la pensée même, il est donc le centre de l'être humain. (p. 436) 

nous sommes - est toujours singulière : «[...] notre rapport à ce qui nous entoure, comme à ce qui nous est transmis, est d'abord un phénomène de compréhension " (Zarader, [s. d.], p. 1). Ce phénomène est un phénomène de saisie du sens, qui configure autant qu'il est configuré en nous par un réseau de significations meuble, qui évolue avec l'histoire de chacun: son expérience, son imaginaire, ses connaissances, son projet, etc., un réseau de significations dynamique, qui fait de la compréhension un phénomène sans cesse renouvelé. 

social et singulier), implique que le sens n'est jamais réitéré à l'identique : «[...] dès que l'on comprend, on comprend autrement» (Gadamer, 1996, p. 318). Il y a donc du nouveau dans la compréhension, mais ce nouveau ne rompt pas avec la tradition.

La puissance créative du langage ne réside donc pas uniquement dans la capacité qu'il offre de coder ou décoder des énoncés inédits - comme le postule la créativité linguistique de Chomsky-; pas non plus uniquement (ni peut-être même prioritairement) dans la " production » (au sens usuel de ce terme, en didactique). Nous ne parlons pas d'un langage "fantôme d'un langage pur » (Merleau-Ponty, 2010, p. 1437), avec lequel on ne fait « rien de plus que remplacer une perception ou une idée par un signal convenu qui l'annonce, l'évoque ou l'abrège » (ibid.). Au contraire, pour percevoir l'aspect poḯtique - étymologiquement : créatif - du langage, il faut insister sur la façon dont « chaque individu [...] se forme dans le sein de la même nation ${ }^{10}$ une langue à part, attache des idées autrement modifiées aux mêmes mots, et attire insensiblement le langage commun dans ce qu'il y a de plus essentiel, dans les nuances les plus intimes de la pensée et du sentiment » (Humboldt, 2000, p. 56). Ce lien singulier des idées aux mots, ce transport du langage commun dans les sphères de l'intime, constituent, à proprement parler, le processus de compréhension, tel qu'ébauché plus haut ${ }^{11}$.

\subsection{Faux amis et différences}

Choisissons donc le terme de poïesis pour éviter toute confusion, et prenons le temps d'énoncer ce qui la distingue de la créativité telle que nous l'avons comprise à travers les textes étudiés pour alimenter la première partie de cet article.

1. La poïesis est toujours perceptive avant d'être productive; la poïesis intervient au niveau de l'expérience que l'on a du monde, dans un processus qui lie perception, compréhension et imaginaire. Fortement liée au langage et aux langues (cf. Humboldt et « la vision du monde » - Weltansicht), elle advient déjà en dehors de toute production tangible, au moment, par exemple, où l'odeur d'une madeleine fait surgir chez Marcel Proust tout le monde de l'enfance, avant même qu'elle ne se manifeste de nouveau dans l'écriture de l'auteur.

2. La corporéité de la poïsis se distingue de celle de la créativité (telle que présentée en 1) : si la poiesis est à comprendre en lien avec son incarnation, sa corporéité, c'est ici dans un corps envisagé comme sensible, historique et social, et non plus comme purement biologique ${ }^{12}$.

3. La poïesis relève d'une nécessité existentielle là où la créativité a une utilité communicative et vise l'efficacité ${ }^{13}$ : la compréhension, premier siège de la poïesis, est un phénomène proprement humain, par lequel chacun est au monde avec les autres, y compris dans des moments d'apparente inactivité communicative: sans cette perception/compréhension poïétique (c'est-à-dire créatrice et langagière) du monde, pas de "rapport à ce qui nous entoure » (Zarader, [s. d.], p. 1).

4. La poïesis se produit dans la rencontre altéritaire qui vient bouleverser et renouveler le réseau de significations meuble évoqué plus haut. Le conflit des interprétations qui résulte de cette rencontre génère des « heurts » (Bonoli, 2008), lesquels sont fondateurs dans l'apprentissage (ou, en termes PH, dans l'appropriation).

5. La poïesis, enfin, ne se laisse pas contrôler et ne saurait se fonder sur un quelconque critère d'adaptation. On peut reprendre ici l'opposition effectuée par Bouveresse (1991, p. 45) ${ }^{14}$ lorsqu'il évoque les aspects créateurs du langage: la créativité, telle qu'usuellement entendue en DDdL, relèverait alors, selon ses termes, d'une conception « analytique » du langage et serait, dans ce cadre, "une créativité gouvernée par les règles ». La créativité 
telle que nous l'entendons, la poïesis donc, serait quant à elle d'ordre fondamentalement herméneutique, une créativité qui «change les règles» et qui "devrait dissuader tout théoricien du langage d'essayer d'expliquer l'usage et la compréhension des signes linguistiques par l'action d'un mécanisme quelconque » (ibid.).

\section{Et en DDdL : quelles implications ? quelles perspectives?}

Prendre au sérieux ces éléments en DDdL, c'est penser une créativité qui ne se manifeste pas seulement dans le faire (pas seulement dans une perspective actionnelle), pas seulement dans le tangible (le traçable, le critériable, l'évaluable), mais aussi dans la réception, dans la compréhension, une poïétique, donc. Ainsi, comprendre une langue nouvelle, en écho à ce que l'on est toujours déjà en langue, c'est être créatif, c'est insérer dans un réseau de sens qui nous est propre, de nouvelles significations qui seront ellesmêmes configurées par ce réseau.

Le fond antéprédicatif d'où surgit le langagier, ainsi que le caractère incarné (sensible, historique, singulier) et existentiel de la poïesis engagent à penser, dans ce cadre, l'apprentissage comme une "appropriation" (Castellotti, 2017), au sens où tout apprentissage d'une langue peut devenir apprentissage en langue. L'apprentissage d'une langue correspondrait ici à l'idée d'emmagasiner des objets linguistiques, afin d'être en mesure de "remplacer une perception ou une idée par un signal convenu ${ }^{15}$ " pour reprendre les termes de Merleau-Ponty. Quant à l'apprentissage en langue, il permettrait de souligner l'idée selon laquelle, dans l'apprentissage, s'opère une appropriation de sens qui, d'étrangers, adviennent en propre à celui qui apprend ; à celui qui, dans son effort d'apprentissage, se (trans-)forme.

Les démarches "artistiques", si elles sont envisagées sous l'angle de l'expérience, constituent alors, plutôt qu'un détour, un chemin - parmi d'autres, sans doute - pour favoriser cette appropriation (poïétique), en ce qu'elles offrent la possibilité d'une véritable rencontre altéritaire.

\subsection{La possibilité d'une œuvre}

Nous avons jusqu'ici insisté sur la dimension poïétique du langage par la médiation duquel nous comprenons le monde auquel nous sommes. Comme nous l'avons déjà dit, cette dimension implique que le langage fasse davantage que d'apposer un son sur un référent qui lui préexiste: chaque langue, dans ses dimensions à la fois partagée et toujours aussi singulière, fait surgir un monde de ce qui resterait sans elle indistinct. Les langues sont ainsi à la fois interprétantes (en ce qu'elles découpent le monde en catégories et l'articulent en une syntaxe) et interprétées (en ce qu'elles ne se rencontrent qu'à travers les discours, par le biais donc de leur incarnation). Ces deux dimensions peuvent être mobilisées dans le cadre de la DDdL, à travers, notamment, la possibilité offerte à ceux qui sont venus apprendre, de faire l'expérience d'une œuvre, car «l'œuvre puise dans le réel pour nous le rendre autrement, et nous permettre de l'habiter de façon nouvelle. Elle n'est donc pas close sur elle-même. Elle est médiatrice du monde " (Zarader, [s. d.], p. 2). 

dans la perspective PH que nous avons choisi d'adopter, «le sens (qu'il s'agit de saisir dans la compréhension) n'est pas donné une fois pour toutes (dans l'œuvre ou dans le texte), il est essentiellement en devenir, car il ne s'accomplit que dans la réception » (Zarader, [s. d.], p. 1).

a lire un texte, le recevoir, c'est faire surgir un monde, au prisme du texte, et en écho à l'expérience du lecteur. Cette expérience de l'œuvre est « une possibilité de l'œuvre» (Zarader, [s.d.], p.4), chaque fois renouvelée. Autrement dit, l'« appropriation au présent [de l'œuvre d'art] est constitutive de l'être de l'œuvre » ( ibid., p. 6), à rebours d'une conception de la créativité pensée prioritairement à partir de l'action. ce qu'elles évoquaient aux élèves, et sur la nature de ce qui était représenté (écriture ou dessin). Ces discussions ont amené les élèves à puiser dans leurs diverses expériences, dans leurs imaginaires, dans leurs connaissances de manière à pouvoir étayer leurs interprétations. La présentation d'associations « libres » était aussi possible. œuvre, d'en expliciter leur réception. Au-delà de l'intention de l'auteur, ils ont recréé un sens à cette œuvre dont ils ont fait l'expérience.

Après cela, les adolescents ont été invités à s'inspirer de ces images en les envisageant comme une suite à laquelle ils devaient proposer un troisième élément. Leur attention a été attirée sur le fait qu'une fois leur proposition créée, ils seraient interrogés sur leurs choix, les motivations et intentions de leurs œuvres. L'introduction de cette dimension réflexive est importante dans la mesure où elle ne cantonnait pas la séance d'arts plastiques au faire. Par cette entreprise de double traduction - d'une compréhension en œuvre et d'une œuvre en discours - ils ont explicité - parfois - de quelle manière ils ont intégré à leur réseau de significations des modifications issues des discussions autour des images qui leur avaient été soumises.

Tous ces échanges ont eu lieu principalement en français, mais aucune langue n'était interdite durant les ateliers. Au-delà du «bain linguistique » qu'ils n'ont pas manqué de constituer, le principal attrait didactique de ces ateliers est, à notre sens, lié à la rencontre altéritaire qu'ils ont (pour certains élèves) permise : rencontre avec l'œuvre, mais rencontre(s) aussi autour de l'œuvre.

\subsection{Perspectives}

Nous avons construit cet article de manière à mettre en regard deux acceptions de la notion de «créativité » en DDdL. La première, dominante en DDdL aujourd'hui, met 
l'accent sur la production, la seconde - que nous avons choisi de nommer poïesis (plutôt que créativité) pour plus de clarté - sur la réception. Une réception poïétique qui vise à saisir le sens en le reconfigurant toujours, une réception qui se fait toujours dans la rencontre, telle qu'envisagée par Romano (1999) :

Que serait, en effet, une rencontre, si elle ne mettait l'advenant en demeure de se comprendre lui-même autrement à partir de possibles reconfigurés? Que serait une rencontre, si elle ne faisait chanceler nos certitudes, nos habitudes, si elle ne détruisait toute possibilité d'être habitué à quoi que ce soit en nous faisant douter de tout, y compris et d'abord de notre propre capacité à accueillir celui ou celle dont le destin n'a pas seulement croisé, mais altéré et bouleversé le nôtre ? (p. 232)

Cette réception, toujours reconfigurante, remet en question la nouveauté comme critère définitoire de la créativité, dans le sens où la réception n'est, ici, jamais réitérée à l'identique. La corporéité pourrait sembler être un point commun à ces deux approches, mais qui doit en fait être relativisé : la première acception se soucie prioritairement de la dimension biologique du corps, là où la seconde s'intéresse à son caractère sensible et historial.

C'est encore dans les visées de leur mobilisation que ces notions diffèrent: l'une vise l'apprentissage, l'autre l'appropriation (Castellotti, 2017): dans le premier cas, la créativité est une façon d'agir en détour pour mieux s'adapter à un nouveau contexte ; dans le second, la poïesis est au fondement du projet didactique, en tant que mode d'être au monde : en tant que partie prenante de l'appropriation (Lorilleux, 2015), elle constitue en effet un ressort d'émancipation. Dans un cas, la didactique sort indemne de la créativité : elle conserve la maitrise de ses objectifs, des moyens qu'elle se donne pour y parvenir et mobilise l'art comme un détour, un émollient (utile, donc) qui faciliterait l'accès au savoir. Dans l'autre, la didactique se trouve transformée à coeur par cette acception forte de la créativité - la poïesis - et notamment par la non-contrôlabilité dont celle-ci procède. L'expérience herméneutique, en effet, ça ne se commande pas: en premier lieu, l'enseignant ne sait pas s'il ressortira quelque chose de la fréquentation de l'œuvre qu'il propose. En second lieu, s'il en ressort quelque chose, il est impossible d'anticiper sur ce qui en ressortira. S'il peut, comme l'y invite Jacotot, toujours confronter l'élève à la triple question "Que vois-tu? Qu'en penses-tu? Qu'en fais-tu?» (Rancière, 1987, p. 42), l'enseignant ne peut jamais savoir à l'avance si et comment cette œuvre sera intégrée au réseau de significations singulier de chacun. Il lui faudrait donc, dans cette perspective, se résoudre à "accompagner sans savoir où " (Lorilleux \& Castellotti, sous presse; Lorilleux, 2015). Démarche que d'aucuns pourraient qualifier d'in-utile, mais qui nous semble, quant à nous, essentielle.

\section{BIBLIOGRAPHIE}

ADEN, Joëlle. (2009). La créativité artistique à l'école : refonder l'acte d'apprendre. Synergies Europe , 4, 173-180.

BenKirane, Reda. (2006). La complexité, vertiges et promesses. Paris : Le Pommier. 
BESSE, Henri. (2000). Propositions pour une typologie des méthodes de langues (Thèse de doctorat). Université Paris 8 - Vincennes-Saint-Denis.

BONOLI, Lorenzo. (2008). Lire les cultures : la connaissance de l'altérité culturelle à travers les textes. Paris : Kimé.

Botella, Marion, NeLSON, Julien \& ZeNASNI, Franck (2016). Les macro et microprocessus créatifs. Dans I. Capron Puozzo (dir.), La créativité en éducation et en formation. Perspectives théoriques et pratiques (p. 33-46). Louvain-la-Neuve : De Boeck.

Bouveresse, Jacques. (1991). Herméneutique et philosophie. Paris : Éditions de l'Éclat.

CAPRON PUOZzo, Isabelle. (2015). La créativité pour des pratiques pédagogiques innovantes.

Communication présentée au colloque « Regards pluridisciplinaires sur la créativité et l'innovation en langues étrangères », Toulouse (11-12 décembre 2015).

CAPRON PuOzzo, Isabelle (dir.). (2016). La créativité en éducation et en formation. Perspectives théoriques et pratiques. Louvain-la-Neuve : De Boeck.

CASTEllotTI, Véronique. (2017). Pour une didactique de l'appropriation. Diversité, compréhension, relation. Paris : Didier.

CAstellotti, Véronique, Debono, Marc \& Huver, Emmanuelle (2017). Une «tradition de l'innovation » ? Réflexion à partir du corrélat innovation/créativité en DDdL. Travaux neuchâtelois de linguistique, 65, 113-130. Disponible en ligne sur <www.unine.ch/files/live/sites/tranel/files/ Tranel/65/113-130_Varia_def.pdf>.

Сномкку, Noam. (1982). A Note on the Creative Aspect of Language Use. The Philosophical Review, 3, 423-434.

DASTUR, Françoise. (2014). Penser ce qui advient. Paris : Les petits Platons.

DEBono, Marc. (2016). Deux grandes conceptions de la réception (et leurs places respectives en sociolinguistique francophone). Glottopol, 28. Disponible en ligne sur <http://glottopol.univrouen.fr/telecharger/numero_28/gpl28_08debono.pdf>.

DRACH, Margaret. (1981). The Creative Aspect of Chomsky's Use of the Notion of Creativity. The Philosophical Review, 1, 44-65.

GADAMER, Hans-Georg. (1996). Vérité et méthode [1960]. Paris : Seuil.

HUMBOLDT, Wilhelm von. (2000). Sur le caractère national des langues et autres écrits sur le langage. Paris : Seuil.

JOSEPH, John E. (2004). Créativité linguistique, interprétation et contrôle de l'esprit selon Orwell et Chomsky. Dans P. Sériot \& A. Tabouret Keller (dir.), Le discours sur la langue sous les régimes totalitaires, Cahiers de l'ILSL, 17, 81-92.

JOSEPH, John E. (2011). Théories et politiques de Noam Chomsky. Langages, 182, 55-67.

LORILLEUX, Joanna. (2014). Le paradoxe de la classe, ou l'expérience formative à l'épreuve de la catégorisation. Glottopol, 23, Inaccessibles, altérités, pluralités : trois notions pour questionner les langues et les cultures en éducation, 116-133.

LORILLEUX, Joanna. (2015). Écritures transformatives. Quand des élèves allophones deviennent auteurs, ou : de l'appropriation à l'émancipation (Thèse de doctorat). Université François Rabelais, Tours. LORILLEUX, Joanna \& CASTELLOTTI, Véronique. (Sous presse). Face à l'idéologie uniciste, une éducation émancipatrice. Dans E. Suzuki, A. Potolia, S. Cambrone, N. Carré, J.-O. Kim, L. Ouvrard 
\& R. Zhang-Fernandez (dir.), Penser la didactique du plurilinguisme et ses mutations : politiques, idéologies, dispositifs.

LUBART, Todd. (2003). Psychologie de la créativité. Paris : Armand Colin.

MERLEAU-PonTY, Maurice. (2010). Euvres. Paris : Gallimard.

Morin, Edgar \& TAUBIRA, Christiane. (2017). Pour une poétique des civilisations. Le Monde, 29 juillet. Disponible en ligne sur <http://www.lemonde.fr/festival/article/2017/07/29/edgarmorin-et-christiane-taubira-pour-une-poetique-descivilisations_5166380_4415198.html\#JkqcqFl4h3H4BJty.99>.

PICCARDO, Enrica. (2016). Créativité et complexité : quels modèles, quelles conditions, quels enjeux ? Dans I. Capron Puozzo (dir.), La créativité en éducation et en formation. Perspectives théoriques et pratiques (p. 47-64). Louvain-la-Neuve : De Boeck.

Puozzo CAPRON, Isabelle. (2014). Pour une pédagogie de la créativité en classe de langue. Réflexion théorique et pratique sur la triade créativité, émotion, cognition. Voix plurielles, 11(1), 101-111.

RANCIÈRE, Jacques. (1987). Le Maître ignorant. Paris : Fayard.

ROBILLARD, Didier DE. (2009). Ce que « comprendre » pourrait bien vouloir dire ? Langage et société, $130,125-136$.

ROBILLARD, Didier DE (dir.). (2017). Épistémologies et histoire des idées sociolinguistiques, Glottopol, 28. Disponible en ligne sur <http://glottopol.univ-rouen.fr/numero_28.html>.

Romano, Claude. (1999). L'événement et le temps. Paris : PUF.

SOFIA, Gabriele. (2016). Embodied creativity. Formation de l'acteur et neurosciences de la créativité. Dans I. Capron Puozzo (dir.), La créativité en éducation et en formation. Perspectives théoriques et pratiques (p. 93-104). Louvain-la-Neuve : De Boeck.

THouARD, Denis. (2000). Présentation - L'embarras des langues. Dans W. von Humboldt, Sur le caractère national des langues et autres écrits sur le langage. Paris : Seuil.

TRABANT, Jürgen. (2014). Pourquoi Humboldt ? Dans S. Archaimbault, J.-M. Fournier \& V. Raby (dir.), Penser l'histoire des savoirs linguistiques (p. 573-585). Lyon : ENS Éditions. Disponible en ligne sur <http://juergen-trabant.de/wp-content/uploads/2012/10/Trabant-2014e-Pourquoi.-pdf.pdf>.

ZARADER, Marlène. (s. d.). Compréhension et interprétation dans l'herméneutique de Gadamer. Disponible en ligne sur <https://disciplines.ac-montpellier.fr/philosophie/sites/philosophie/ files/fichiers/2006/zarader_gadamer.pdf>.

\section{NOTES}

1. Certains considérant comme de la créativité ce que d'autres interprètent comme des formes corrompues, abâtardies de la langue.

2. Chomsky, cité par Drach (1981, p. 60) : «Une personne qui connait une langue maitrise un système de règles qui assigne un nombre fini de signifiés et de signifiants à un nombre infini de phrases possibles. » (Notre traduction)

3. Chomsky, cité par Drach (1981, p. 60) et par Joseph (2004, s. p. et 2011, p. 57) : «Une personne linguistiquement mûre peut produire dans sa langue une nouvelle phrase quand l'occasion le demande, que d'autres personnes peuvent comprendre immédiatement, bien qu'elle leur soit non moins nouvelle. L'essentiel de notre expérience linguistique, en parlant et en écoutant, est faite de phrases nouvelles. Une fois que nous avons maîtrisé une langue, la gamme de phrases 
avec lesquelles nous pouvons opérer couramment et sans difficulté ou hésitation est si vaste qu'en pratique, [...] on peut la considérer comme infinie. » (Traduction de Joseph, 2011)

4. Il serait intéressant de réfléchir aux raisons de cette amnésie ou de cet effacement. On pourrait supposer que le fait de référer à Chomsky dans des travaux qui s'inscrivent très explicitement dans des perspectives émergentistes paraitrait particulièrement incongru, voire inacceptable.

5. Entendu ici dans son sens large de "inscription dans les sciences cognitives ", sans distinction entre les différents courants qui le composent.

6. Et, en amont, déculturalisée, comme l'évoquait déjà Besse (2000, p. 164-165).

7. Pour des raisons de place, nous ne ferons ici qu'exposer les thèses principales de ces courants. Pour une présentation des arguments qui le sous-tendent en sociolinguistique et en DDdL, et notamment au prisme de la notion de diversité, cf. Castellotti (2017) et Robillard (2016).

8. Ou, plus exactement, au sensible, y compris dans ses dimensions historiales - ce qui relève d'une autre conception de la corporéité que celle, essentiellement physiologique développée dans le cadre de l'enaction.

9. L'emploi de cette expression permet incidemment d'illustrer notre propos : il est évident que national revêt aux yeux de lecteurs contemporains un sens tout autre que celui auquel il renvoyait durant le premier quart $\mathrm{du} \mathrm{XIX}^{\mathrm{e}}$ siècle, que nous pourrions peut-être traduire aujourd'hui par « culturel».

10. Dans le sens évoqué dans la note 9 de bas de page.

11. Outre l'ouvrage de Gadamer (1960), cf. aussi Robillard (2009), Lorilleux (2014), Debono (2016) et Castellotti (2017).

12. Le traitement $d u$ « sensible » sous l'angle, principalement, des émotions est symptomatique de cette biologisation.

13. Cf. par exemple la notion, très prégnante actuellement, de «sentiment d'efficacité personnelle ». À l'inverse, la poïesis, telle que nous la mobilisons ici rejoint, sur ce point en tout cas, la poétique défendue par Morin et Taubira (2017), en ce qu'ils insistent sur son caractère prioritairement politique. Elle ne relève pas uniquement - ni essentiellement - du culturel et de l'artistique, mais est à comprendre plus largement comme un « rapport à la pensée, à la parole et à la relation", en dehors de tout «rapport quantophrénique, de quantification et de vérification ".

14. Et ce, même si Bouveresse se situe lui-même explicitement du côté de la philosophie analytique.

15. Autrement dit avoir un usage utilitaire de la langue, ce qui n'est bien sûr pas à négliger.

\section{RÉSUMÉS}

La notion de créativité, telle que souvent conceptualisée en didactique/didactologie des langues (DDdL), est ici mise en discussion : les définitions de la notion les plus fréquemment rencontrées en DDdL sont rappelées, puis mises en regard avec les principes d'un autre univers de référence, la phénoménologie herméneutique, afin d'envisager une autre acception possible de la créativité alors nommée poiésis. Sont enfin discutées les implications pour la DDdL qui découlent de la mobilisation de l'une ou l'autre acception de la notion.

The notion of creativity, in the way it is commonly conceptualized in language teaching and didactology (LTD), is debated in this paper. After reminding the most frequent definitions of the 
notion in LTD, we will contrast them with the principles of another reference universe: phenomenology-hermeneutics, so as to consider an alternative understanding of creativity thereby called poiesis. That contrast will help discuss the implications of the use of various understandings of the notion in language teaching.

INDEX

Keywords : creativity, poïesis, artistic approaches, experience, appropriation, reception

Mots-clés : créativité, poïesis, démarches artistiques, expérience, appropriation, réception

\section{AUTEURS}

EMMANUELLE HUVER

Université de Tours, EA 4428 Dynadiv

\section{JOANNA LORILLEUX}

Université de Tours, EA 4428 Dynadiv 